\title{
PASSIVE INFRARED DETECTOR FOR SECURITY SYSTEMS DESIGN, ALGORITHM OF PEOPLE DETECTION AND FIELD TESTS RESULT
}

\author{
M. KASTEK, H. MADURA \& T. SOSNOWSKI \\ INSTITUTE OF OPTOELECTRONICS, \\ MILITARY UNIVERSITY OF TECHNOLOGY, WARSAW, POLAND
}

\begin{abstract}
The article presents passive infrared (PIR) detectors used for the protection of large objects (buildings, airports, and border) or large areas having larger detection ranges and complex algorithms of signal analysis, upon which the efficiency of detectors' operation is significantly dependent. The construction and principle of operation of PIR detectors of a large detection range is presented. The article also presents the design and principle of operation of a PIR detector of a large detection range. The significant virtue of the described PIR detector is a highly efficient detection of very slowly moving or crawling people.
\end{abstract}

Keywords: passive infrared detector, probability of detection, security systems.

\section{INTRODUCTION}

Passive infrared (PIR) detectors operating in far infrared range $(8-14 \mu \mathrm{m})$ are frequently used in security systems for people detection. Because of their passive operation, it is difficult to detect them and due to suitably chosen spectral range of operation they are low sensitive to disturbances caused by sun radiation. For PIR detectors used in security systems, the pyroelectric sensors of high sensitivity are often used [1,2]. Passive infrared detectors used for the protection of large objects (buildings and airports) or large areas have larger detection ranges and complex algorithms of signal analysis, upon which the efficiency of detectors' operation is significantly dependent.

The essential drawback of the currently available PIR detectors is their low efficiency of detection of slowly moving or crawling people. The efficiency of slowly moving objects is low because the changes in thermal radiation from these objects are close to thermal fluctuations of a background [3]. Moreover, to detect slowly moving (or crawling) people, the lower frequency limit of a transmission band of a PIR detector has to be close to zero. When this condition is fulfilled, an increase in low frequency noises is observed. Thus, detection algorithms must be different from those used for a typical PIR detector. To detect crawling people, a higher number of sensors should be applied (more detection zones), which increases a signal-to-noise ratio, because each of the sensors can 'see' a smaller observation area.

Multitude of factors influencing the signal received from a pyroelectric sensor often causes false alarms generated by PIR detectors. Therefore in order to reduce the number of false alarms caused by, e.g. air turbulences or changes in thebackground temperature resulting from sun radiation, the sensors with two active elements are used (two sensors) and an alarm signal is determined by the analysis of a difference of their output signals [4]. Distinguishing a useful signal (coming from an object) from a noise signal (arising as a result of disturbing factors) is a serious recognition problem. Thus, the important task of a PIR detector, especially in an elongated detection zone, is such conversion and analysis of the signal to achieve a relatively high probability of object detection with simultaneous low probability of a false alarm detection [3]. 


\section{CONSTRUCTION AND PRINCIPLE OF OPERATION OF A PIR DETECTOR}

The main elements of a PIR detector are the objective (mirror or refraction one), a set of pyroelectric sensors, and electronic systems (Fig. 1). The sensors convert an optical signal, emitted from the 'being observed' surface, into an electrical signal. This signal is processed in the electronic systems (it is amplified, filtered, and sampled) and next analyzed in a microprocessor system.

The PIR detector presented here detects the crawling people at a distance of $140 \mathrm{~m}$. A high signal-to-noise ratio (SNR) was obtained due to the application of a larger number of pyroelectric sensors, i.e. a larger number of detection zones (channels). The application of a larger number of sensors forces the necessity to develop a complex optical system. The optical system of a PIR detector has to ensure such a position of detection zones (Fig. 2) to avoid the existence of the areas that 'cannot be seen' by a detector [3].

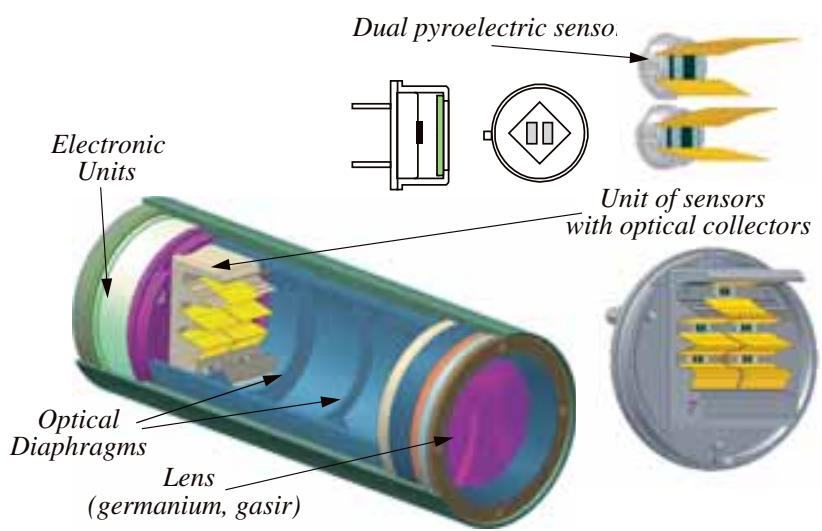

Figure 1: A simplified diagram of a PIR detector and pyroelectric sensors with optical collectors.

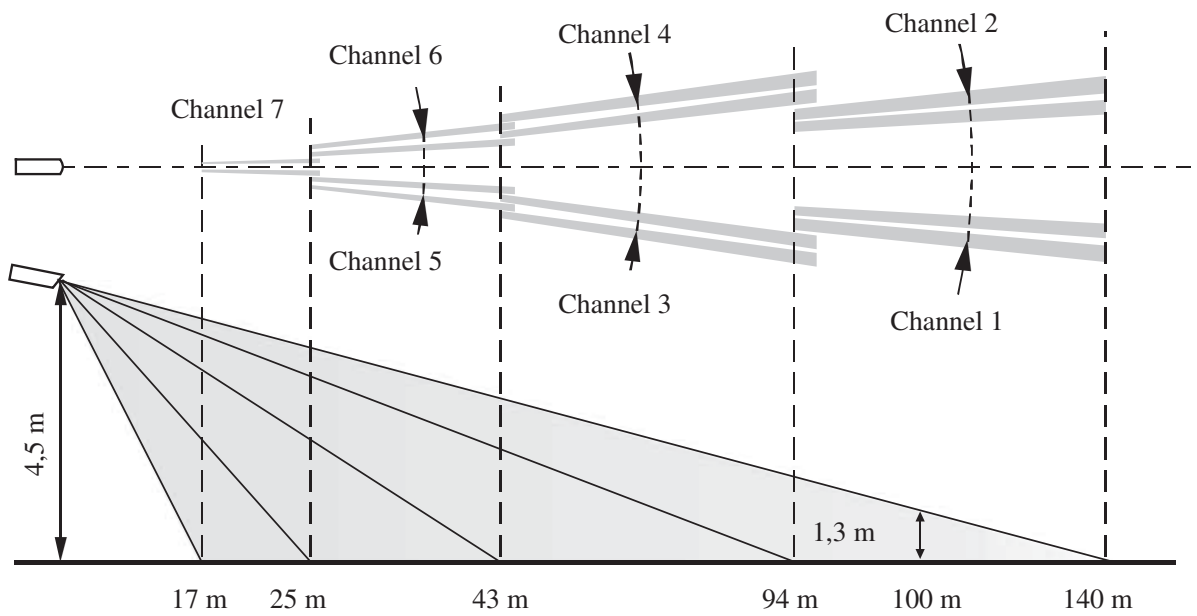

Figure 2: Detection zones of a PIR detector in horizontal and vertical planes. 
Thermal radiations generated by slowly moving and (especially) crawling people are characterized by similar amplitudes of luminance, and the velocities of this luminance change in time, such as fluctuation change of background radiation. The amplitude of a signal from pyroelectric sensors is directly proportional to the velocity of a change in a radiation signal in time (i.e. to the velocity of a moving object). The disadvantageous property of pyroelectric sensors is a voltage drift owing to the changes in ambient temperature (pyroelectric sensor is equipped with a field transistor playing a role of a voltage follower). Thus, to have a sensor efficiently detecting slowly moving people, it is necessary to develop an algorithm eliminating the changes in a signal from a sensor caused by background noise as well as the changes in a signal caused by temperature drift of a pyroelectric sensor.

A simplified diagram of an electronic circuit of a PIR detector is shown in Fig. 3. At ' + ' input of the amplifying stage, a reference voltage is applied, which is obtained from a resistance divider and a digital potentiometer included between the supply voltage and the system's ground.

A system of DC amplifier with regulation (by means of digital electronic potentiometers) of a level of a constant component consists of three operation amplifiers. The first $U_{1 \mathrm{~A}}$ amplifier, because of a significant output resistance of a pyroelectric sensor operates in a follower system and two consecutive ones in the systems reversing a signal phase. The first stage of amplification is realized by the operation amplifier $U_{3 A}$. It is characterized with the signal amplification $K_{1}=-R_{4} / R_{3}$. At its '+' input, a reference voltage is applied, which is obtained from the resistance divider $R_{5}, R_{6}, U_{2 A}$ (digital potentiometer) included between the supply voltage and the system ground [5].

The reference voltage (regulated by means of a digital electronic potentiometer) is used for elimination of a constant component of a signal obtained from a pyroelectric sensor. This component is significantly changed together with temperature changes occurring near a

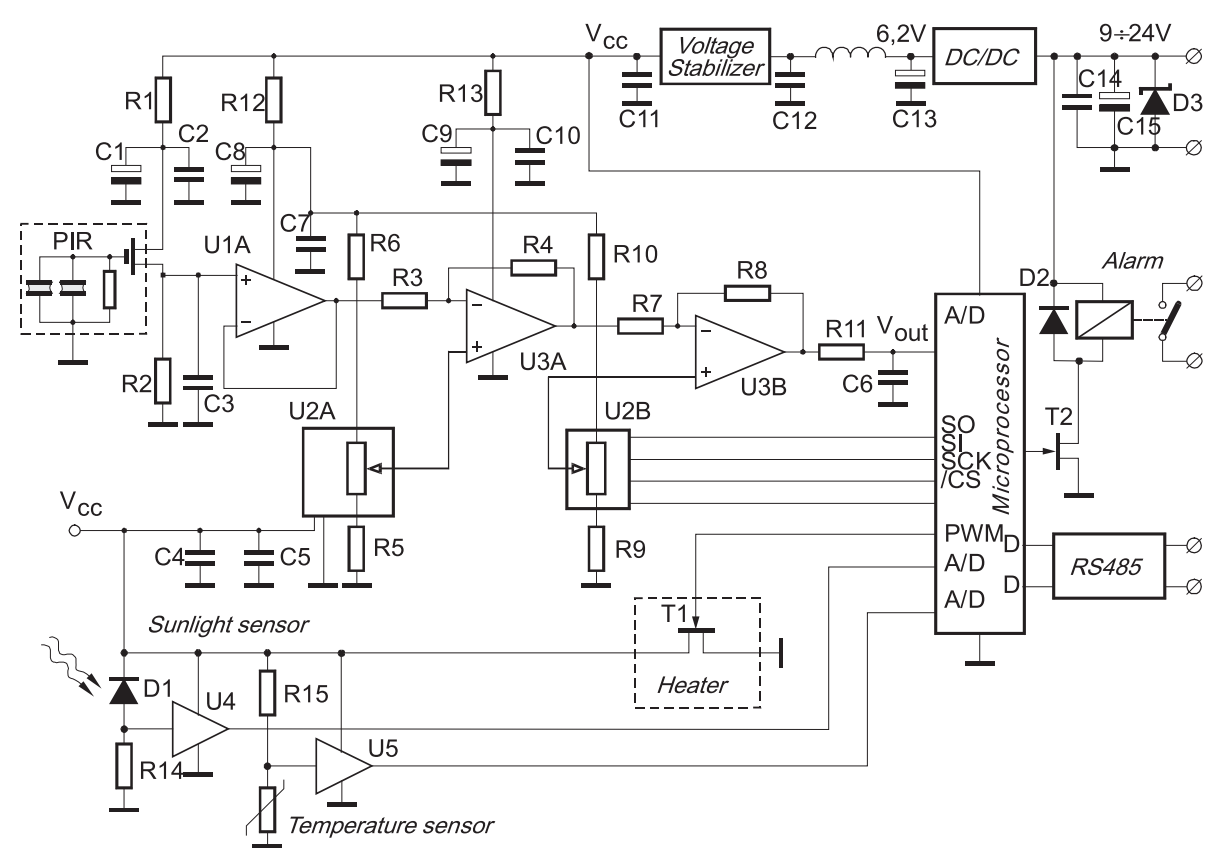

Figure 3: A simplified functional scheme of an electronic system of a PIR detector. 
pyroelectric sensor (because of a change in the output current change of a field transistor dependent on temperature). Next, the signal is delivered to the second amplifying stage of the $U_{3 B}$ operation amplifier, the amplification of which being $K=-R_{8} / R_{7}$. At its ' + ' input, the reference voltage is obtained from the $R_{9}, R_{10}, U_{2 B}$ resistance divider (digital potentiometer). This voltage (regulated by means of a digital electronic potentiometer) protects the amplifier against the saturation state. The output signal is filtered in the filter made from $R_{11}$ and $C_{6}$ elements. The electronic potentiometers, used in a preamplifier, are connected through the SPI series bus with a controlling processor, which on the basis of the output signal values makes the current adequate regulations (change in the value of potentiometer resistances) of the values of reference voltages of particular amplifying stages.

Knowing the $N_{A}$ and $N_{B}$ settings (the number of a selected level) of particular digital potentiometers, their total resistances $R_{A}$ and $R_{B}$, and the values of amplifications of particular $K_{1}, K_{2}$ preamplifier stages, the signal value $V_{O U T}$ at the amplifier output can be calculated as follows:

$$
V_{\text {OUT }}=V_{I N} K_{1} K_{2}-U_{p A} K_{1} K_{2}-U_{p B} K_{2},
$$

where $V_{I N}$ is the input voltage of the first amplifying stage, $U_{p A}$ is the reference voltage of the first amplifying stage, and $U_{p B}$ is the reference voltage of the second amplifying stage.

The values of reference voltages of particular amplifying stages for 8-bit resolution of digital potentiometers settings can be calculated using the following relationship:

$$
\begin{gathered}
U_{p A}=\frac{V_{c c}}{R 6+R_{A}+R 5}\left(R 5+\frac{N_{A}}{256} R_{A}\right), \\
U_{p B}=\frac{V_{c c}}{R 10+R_{B}+R 9}\left(R 9+\frac{N_{B}}{256} R_{B}\right),
\end{gathered}
$$

where $V_{c c}$ is the supply voltage.

The $U_{p A}$ and $U_{p B}$ values are currently matched by a microprocessor such that ensuring adequate dynamics of the amplifier and preventing the saturation state of particular amplifying stages. If the output voltage of the amplifier was measured and analyzed by a microprocessor, which in its operation algorithm considers the shift in levels (potentiometer settings), it can be shown that the voltage calculated (by a microprocessor) can be significantly higher than the supply voltage. It is an important virtue of this system.

In the PIR's electronic system, the system for the measurement of sun radiation intensity was used. The information from this system is taken into account in an intruder detection algorithm [3]. Moreover, a temperature sensor was used in the system. The data from a temperature-measuring system can be applied to switch-on a miniature heater, mounted inside a PIR detector housing. A small increase in the temperature of the PIR detector's inside (in relation to ambient temperature) protects the deposition of water vapor on optical elements (lens, concentrator mirrors, and optical windows of sensors).

Passive IR sensors have specially formed angular characteristics of detection, achieved by the application of appropriate optical elements, placement of PIR detectors on the focal plane (Fig.1), and the use of additional mirrors acting as beam concentrators. Figure 4 shows module pyroelectric detectors with concentrators and electronics board.

On the one hand, the angle of view of a detector can be enlarged by increasing its size or by using several types of optical elements, such as cylindrical field lenses, aplanatic lenses, and flat mirrors. Flat mirrors are the cheapest and easiest to implement, although the resulting increase in angle of view is smaller than that for other solutions. The optical concentrator 

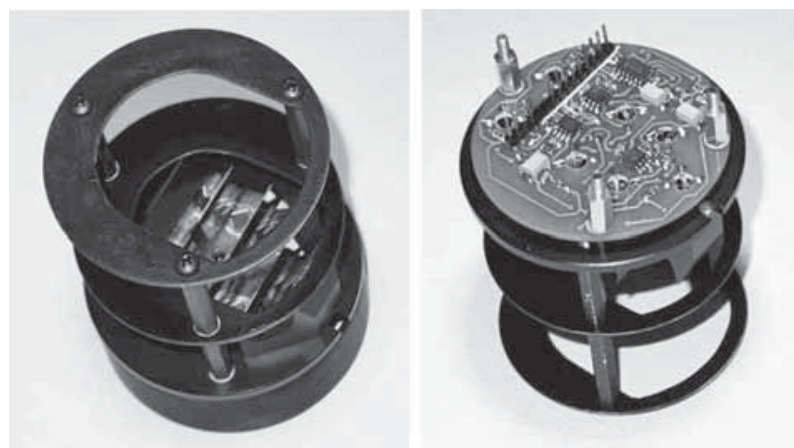

Figure 4: The module pyroelectric detectors with concentrators and electronics board of a PIR detector.

consists of flat mirrors mounted at a certain angle with respect to the detector's optical axis. It is a simple, cost-effective solution that does not introduce additional losses and the resulting angle of view is independent on the wavelength. On the other hand, the resulting increase in the angle of view is limited to several degrees for a single detector.

Optimization of mirrors' placement is a bit complicated task due to a large number of parameters that affect the final efficiency of focusing the radiation on the detectors. These parameters are linear dimensions of a mirror, coordinates of its center, and mirror tilt angle. The angular relations are different for each mirror, depending on a particular observation zone of each detector. The optimization of mirror and detector locations was performed using ZEMAX software. The quality of optical design was verified by examining the relative irradiance of detectors by reflected radiation as a function of angle between the mirror and detector surfaces.

According to numerical calculations, at an angle smaller than 40 degrees all the radiation misses the detector completely. The irradiance increases with the angle, reaching a maximum value at an angle of approximately 70 degrees (Fig. 4).

ZEMAX software makes it possible to calculate the relative irradiance of seven detectors paired up with optical concentrators as a function of target movement in particular detection zones. The results were then verified against real measurement results performed on a laboratory test bed, in order to compare the expected values of parameters with those measured for a real device for all its detection zones [6].

Detection of a person in a detection zone of a PIR detector occurs when a signal level at the output of a detection system, caused by infrared radiation emitted by a person, exceeds a conventional detection threshold. To minimize the probability of false alarms, an adaptation detection threshold 'following' the changing atmospheric conditions should be determined [7].

\section{ANALYSIS OF CHANNEL DETECTION OF A PIR DETECTOR}

The object moving in a detection area, determined by an angle of view of a pyroelectric sensor, produces an electrical signal in it. The spectral distribution (spectral density) of luminance causing the output electric signal can be described as follows:

$$
L_{\lambda}(T, \lambda)=\frac{\varepsilon(\lambda)}{\pi} \cdot M_{\lambda}(T) \cdot \tau_{a}(\lambda) \cdot \tau_{o}(\lambda),
$$

where $\varepsilon(\lambda)$ is the spectral distribution of the emissivity coefficient, $M_{\lambda}(T)$ is the exitance spectral distribution, $\tau_{a}(\lambda)$ is the spectral coefficient of atmosphere transmission, and $\tau_{o}(\lambda)$ is the spectral coefficient of optical systems transmission. 
To simplify the calculations, one can take a constant (independent of wavelength) emissivity coefficient for both the object $\varepsilon_{o}$ and background $\varepsilon_{t}$. An error in calculations caused by using this assumption will be smaller than $1 \%$. A spectral characteristic of transmission of an optical system (germanium lens with a filter and a detector filter) can be described using the following relation:

$$
\tau_{o}(\lambda)=\tau_{s}(\lambda) \cdot \tau_{d}(\lambda)
$$

where $\tau_{s}(\lambda)$ is the spectral coefficient of a germanium lens with antireflection layers and $\tau_{d}(\lambda)$ is the spectral coefficient of transmission of a piezoelectric sensor filter.

Figure 5 shows the spectral characteristics of the transmission coefficient $\tau_{d}(\lambda)$ of the filter of a few pyroelectric sensors.

Integrating eqn (4) for the signal coming from a person of the same, averaged (equivalent) temperature $\overline{T_{O}}$ and for the signal from a background of the average temperature $\overline{T_{B}}$, the following expression for energetic luminances can be obtained:

for a person

$$
L\left(\bar{T}_{O}\right)=\frac{\varepsilon_{o}}{\pi} \int_{\lambda_{1}}^{\lambda_{2}} L_{\lambda}\left(\bar{T}_{O}, \lambda\right) d \lambda \quad\left[\mathrm{W} / \mathrm{cm}^{2}\right],
$$

for a background

$$
L\left(\bar{T}_{B}\right)=\frac{\varepsilon_{t}}{\pi} \int_{\lambda_{1}}^{\lambda_{2}} L_{\lambda}\left(\overline{T_{B}}, \lambda\right) d \lambda \quad\left[\mathrm{W} / \mathrm{cm}^{2}\right] .
$$

Analyzing the case of a detected person, when the sensor 'field of view' is equal to a person surface, the signal at the pyroelectric sensor output is dependent on the difference of temperatures between a person and a background and it is determined as

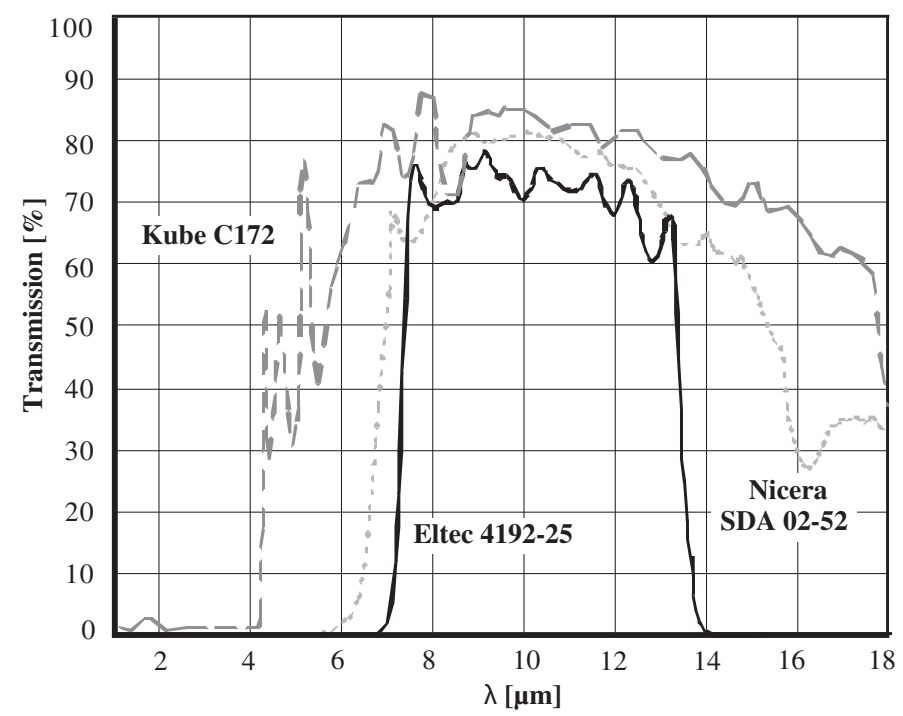

Figure 5: Spectral characteristics of the transmission coefficient $\tau_{d}(\lambda)$ of an optical filter of various pyroelectric sensors. 


$$
S_{O}=k \int_{\lambda_{1}}^{\lambda_{2}}\left[L_{\lambda}\left(\overline{T_{O}}, \lambda\right)-L_{\lambda}\left(\overline{T_{B}}, \lambda\right)\right] d \lambda,
$$

where $k$ is the coefficient dependent on the parameters of pyroelectric sensor, diameter and focal length of the objective, and on emissivity [8-10].

If in the detector a pyroelectric sensor is used, then as the noise signal $S_{B}$, the signal caused by thermal fluctuation of a background can be taken (the change in the background temperature between $T_{B \max }$ and $T_{B \min }$ ). The own detector noise and the amplifier noise is low in comparison with the noise resulting from thermal fluctuation of a background and it can be neglected. Such a noise signal can be described as

$$
S_{B}=k \int_{\lambda_{1}}^{\lambda_{2}}\left[L_{\lambda}\left(T_{B \max }, \lambda\right)-L_{\lambda}\left(T_{B \min }, \lambda\right)\right] d \lambda .
$$

The SNR results from a quotient of the signals given in eqns (7) and (8) and it also depends on the object surface within a field of view of a pyroelectric sensor and it can be calculated using the following relation:

$$
S N R=Z(R) \frac{k \int_{\lambda_{1}}^{\lambda_{2}}\left[L_{\lambda}\left(\overline{T_{O}}, \lambda\right)-L_{\lambda}\left(\overline{T_{B}}, \lambda\right)\right] d \lambda}{k \int_{\lambda_{1}}^{\lambda_{2}}\left[L_{\lambda}\left(T_{B \max }, \lambda\right)-L_{\lambda}\left(T_{B \min }, \lambda\right)\right] d \lambda},
$$

where $Z(R)$ is the covering coefficient and is defined as

$$
\begin{gathered}
Z(R)=\frac{\text { object surface in a sensor's FOV }}{\text { sensor's FOV }}=\frac{A_{o}(R)}{A_{w}(R)}, \\
Z(R)=\left\{\begin{array}{lll}
1 & \text { dla } & \mathrm{A}_{\mathrm{w}} \leq \mathrm{A}_{\mathrm{o}} \\
\frac{A_{o}(R)}{R^{2} \frac{A_{d}}{f^{2}}}=\frac{f^{2} A_{o}(R)}{R^{2} A_{d}} & \text { dla } & \mathrm{A}_{\mathrm{w}}>\mathrm{A}_{\mathrm{o}},
\end{array}\right.
\end{gathered}
$$

where $A_{o}$ is the object surface in a field of view of the sensor, $A_{w}$ is the field of view of the sensor, $A_{d}$ is the sensor surface, $R$ is the distance between the object and the PIR detector, and $f$ is the focal length of an objective.

For short distances, the field of view of a pyroelectric sensor is smaller than the surface of the being observed object and the whole object is contained only for a certain distance in the field of view of a pyroelectric sensor. For larger distances, the value $Z(R)$ decreases faster because the object surface is still constant and the sensors' field of view increases. The change in SNR as a function of a distance between a person and a PIR detector was determined taking into account the difference between the person and background temperatures $\Delta T=\overline{T_{O}}-\overline{T_{B}}$ and the results are shown in Fig. 6.

\section{METHOD OF ANALYSIS OF A PIR DETECTOR SIGNAL}

Detection of a person in a detection zone of a PIR detector occurs when a signal level at the output of a detection system, caused by infrared radiation emitted by a person, exceeds a 

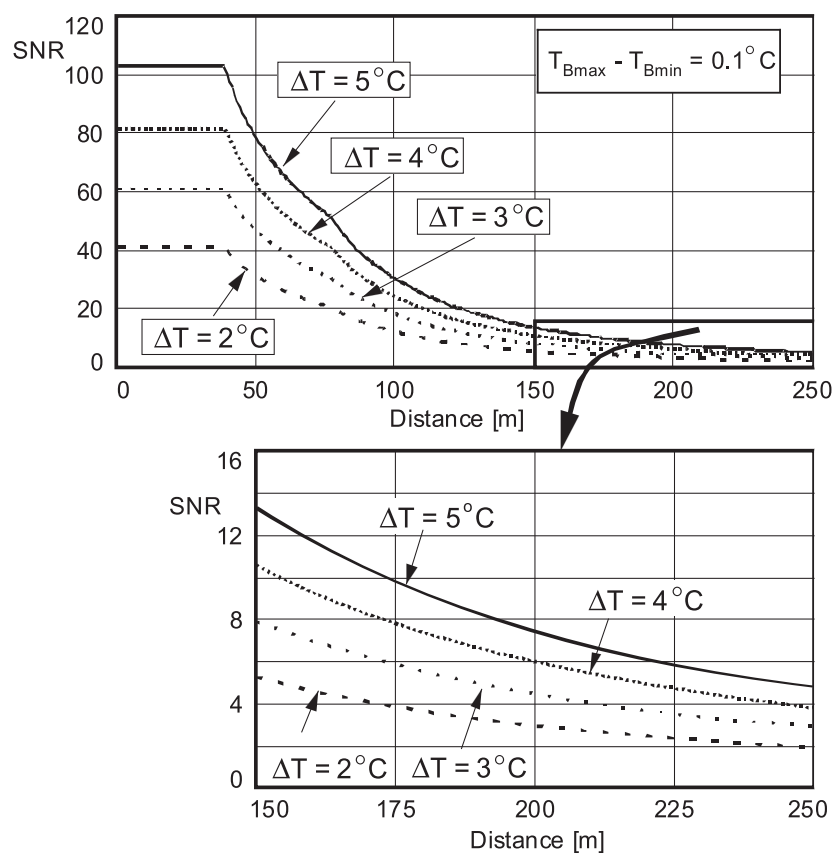

Figure 6: Calculated change in signal-to-noise ratio as a function of a distance between a person and a PIR detector.

conventional detection threshold. To minimize the probability of false alarms, an adaptation detection threshold 'following' the changing atmospheric conditions should be determined $[5,7,9]$.

An analysis of the signal, caused by a moving person and background existing in the field of view of a detector, is made in a microprocessor system [11]. The signal from a detection system is sampled and the voltages of the subsequent signal samples $S_{D}$ are added. Then, a value of such a signal determines eqn (11)

$$
S_{\text {raw }}(i)=\sum_{j=i}^{i-N_{s}-1} S_{D}(j),
$$

where $S_{\text {raw }}(i)$ is the $i^{\text {th }}$ sample of the total signal and $N_{s}$ is the number of added samples.

This signal is a numerical value determined by a microprocessor on the basis of the readout from analog-digital converter and the set value of a digital potentiometer (Fig. 3), which was averaged for 20 -ms period.

An average value of a signal for $N$ samples (Fig. 7) is determined according to the following equation:

$$
\bar{S}_{\text {raw }}(j)=\frac{1}{N} \sum_{i=j-N+1}^{j} S_{\text {raw }}(i),
$$

where $N$ is the number of samples required for the determination of the level of a constant component and $\bar{S}_{\text {raw }}(i)$ is the average value determined for the $i^{\text {th }}$ moment (the $i^{\text {th }}$ sample). 


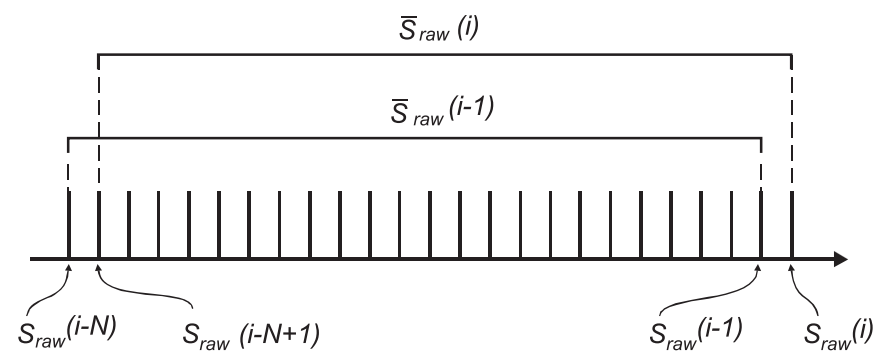

Figure 7: Notations used in a signal analysis method.

Instantaneous deviation from an average value (being an instantaneous level of a constant component) is determined as follows:

$$
R(i)=S_{\text {raw }}(i)-\bar{S}_{\text {raw }}(i)
$$

A central moment of the first row for a set of the parameters $\left\{S_{\text {raw }}(i)\right\}$ can be determined as an instantaneous average value of deviations from the average using the following relation:

$$
\bar{R}(i)=\frac{1}{C} \sum_{j=i-C+1}^{i} R(j)=\frac{1}{C} \sum_{j=i-C+1}^{i}\left[S_{\text {raw }}(j)-\bar{S}_{\text {raw }}(j)\right],
$$

where $C$ is the number of signal samples for which a correction is determined.

Next, a signal component is determined using the following equation:

$$
S(m)=\frac{1}{M} \sum_{i=m-M+1}^{m}\left|S_{\text {raw }}(i)-\bar{S}_{\text {raw }}(i)-\bar{R}(i)\right|
$$

where $M$ is the number of samples in the process of averaging for the $k^{\text {th }}$ set of algorithm parameters, the average value $\bar{S}_{\text {raw }}$ is the local reference level and $\bar{R}$ is the correction resulting from the long-lasting tendency of a signal change.

Finally, the equation allowing for the determination of a signal component takes the following form:

$$
S(m)=\frac{1}{M} \sum_{i=m-M+1}^{m}\left|S_{\text {raw }}(i)-\frac{1}{N} \sum_{j=i-N+1}^{i} S_{\text {raw }}(j)-\frac{1}{C} \sum_{j=i-C+1}^{i}\left[S_{\text {raw }}(j)-\bar{S}_{\text {raw }}(j)\right]\right| .
$$

For a particular case, when $N=C$, the above equation becomes

$$
S(m)=\frac{1}{M} \sum_{i=m-M+1}^{m}\left|S_{\text {raw }}(i)-\frac{2}{N} \sum_{j=i-N+1}^{i} S_{\text {raw }}(j)+\frac{1}{N^{2}} \sum_{j=i-2 N+1}^{i} S_{\text {raw }}(j)\right| .
$$

A signal for further analysis constitutes the weighted sum of signals calculated for each set of parameters according to the following relation:

$$
S(m)=\sum_{k=1}^{K} w_{k} S_{k}(m)
$$

where $w_{k}$ is the coefficient of a signal component. 
A signal, subjected to the analysis referred to objects (intruders) detection, is determined using the following relation:

$$
S^{*}(m)=G \cdot S^{2}(m),
$$

where $G$ is the amplification (constant value) defined for each PIR detector.

A value of an alarm threshold is adaptively determined as

$$
T_{k}=A \cdot \frac{1}{K} \sum_{i=k}^{K+k-1} S_{i}+B \cdot f\left(p_{S}\right)+C,
$$

where $A$ is a constant value, determining the intensity of influence of previous samples of a signal on the value of a detection threshold, $K$ is the number of samples used for averaging process, $C$ is a parameter (constant), the value of which is dependent on the parameters that are set by a user, $B$ is a constant value determining the intensity of influence of sun lighting on the detection threshold value, and $f\left(p_{S}\right)$ is the function dependent on the level of the sun lighting $p_{S}$.

PIR detector generates the alarm signal when the amplitude of the signal read form a detector of the given detection zone (channel) is higher than the adaptively determined alarm threshold. It means that an alarm signal is generated by a PIR detector in the case when the following inequality is fulfilled:

$$
T_{k}>S_{k}^{*}
$$

One the most serious problems of a PIR detector operation is to keep high detection probability at low probability of a false alarm detection. At night, the level of thermal noises of a background is significantly lower than that during the day. Thus, in an IR sensor, switching the IR sensor in day and night modes was used by applying the data from a sensor of a sun lighting level [3, 9].

Passive PIR sensor was made as an element of security system of special, valuable object. It has seven detection zones without blind spots and sophisticated method of intruder detection, capable of finding slowly moving or even crawling people. In order to test its operation and to assess the efficiency of the implemented signal processing method, the measurements of various parameters were performed, both under laboratory conditions and during field tests.

\section{RESULT OF INVESTIGATIONS OF THE METHOD USED IN A PIR DETECTOR}

During the measurements, carried out under various metrological conditions, the data were registered allowing for checking the correctness of algorithm operation, i.e. of a detection threshold determination made in a microprocessor system. The results of signals registration and the calculated values of detection thresholds were registered using the software developed for communication between a PIR detector and a computer. This software provides registration of signals from particular detection zones (channels) and registration of signals from particular stages of signal analysis. Figure 8 shows the test field of a PIR detector.

Figure 9 presents selected records of signals from a PIR detector, which were produced due to a crawling person in an inspection zone. The presented results illustrate a change in the signal in the detector channels caused by a crawling person and adaptive change in the detection threshold resulting from the change in the analyzed signal.

The investigation results presented in the above figures confirmed proper operation of a detector, especially correctness and high efficiency of signal analysis method. Figure 9 shows 

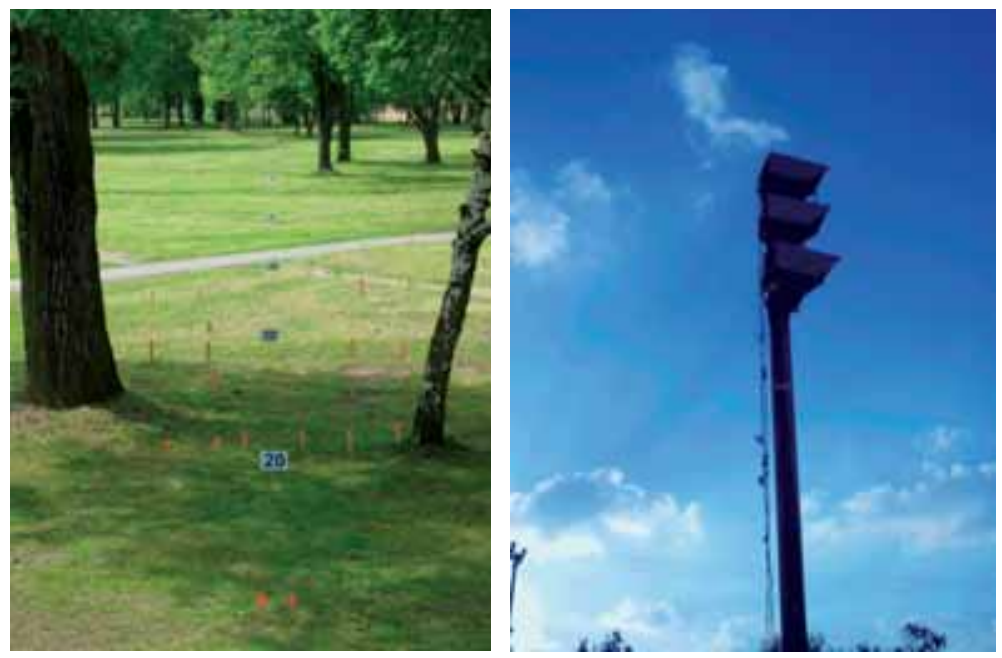

Figure 8: The test field and PIR detector during the tests.

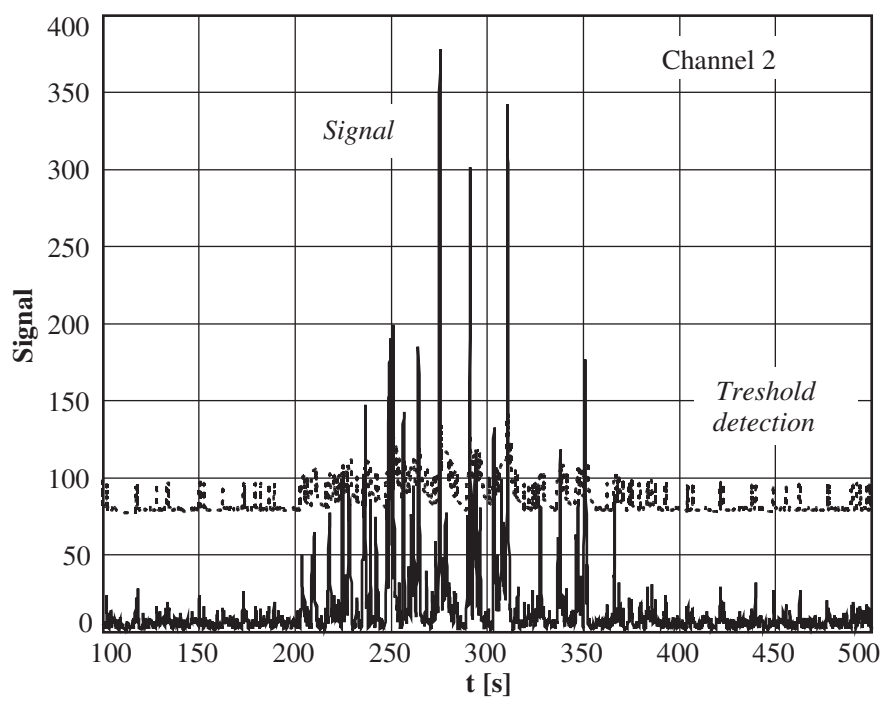

Figure 9: Signal in detection channel 2 of a PIR detector (ambient temperature $26^{\circ} \mathrm{C}$, velocity of a crawling person $0.1 \mathrm{~m} / \mathrm{s}$, distance $140 \mathrm{~m}$ ).

a detection threshold following thermal changes in the background, which, for this measuring case, were caused by sun radiation.

Figure 10 presents the recorded changes in the signal in detection channel 1 of a PIR detector caused by a person walking with a velocity of $1 \mathrm{~m} / \mathrm{s}$, which proves the universality of the used algorithm of a signal analysis allowing for the detection of very slowly or very quickly moving people. 


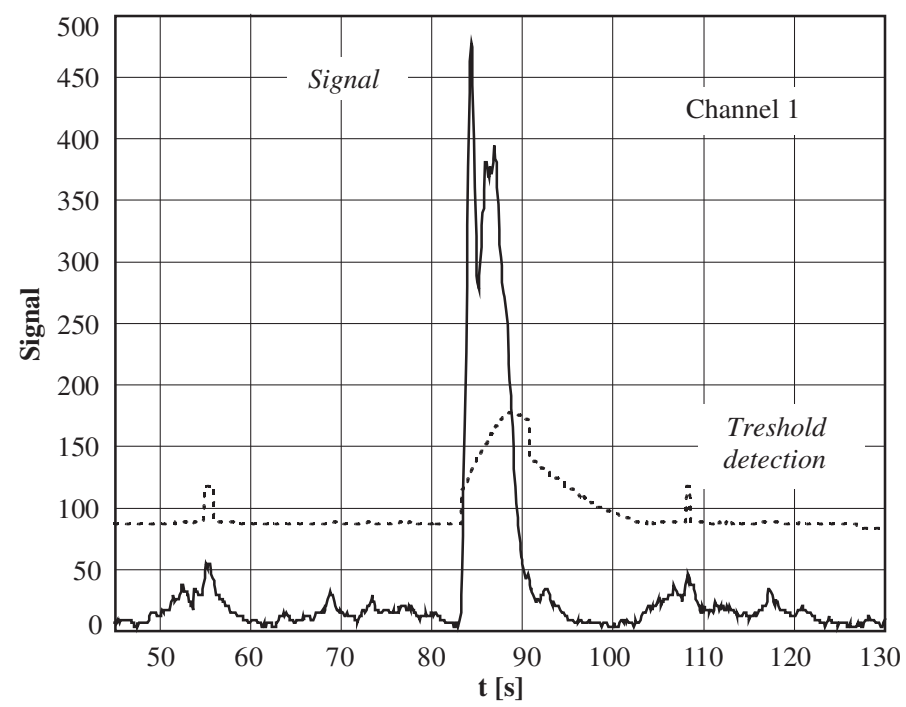

Figure 10: Signal in detection channel 2 of a PIR detector registered at night (ambient temperature $25^{\circ} \mathrm{C}$, velocity of a moving person $1 \mathrm{~m} / \mathrm{s}$, distance $140 \mathrm{~m}$ ).

The efficiency of a signal analysis method, the aim of which is to determine a threshold value of a signal allowing for object detection, can be confirmed by adequately high probability of object detection and a low probability of a false alarm.

\section{DETERMINATION OF A PERSON DETECTION PROBABILITY}

A signal coming from an object is disturbed with the noise generated by a background and electronic systems of a PIR detector. It can be assumed that the noise at the detector output is a white noise, while instantaneous values of voltages can be described with a normal distribution with the expected value $\overline{x_{N}}$ equal to zero and the standard deviation $\sigma_{N}$.

For a signal with Gaussian distribution of probability density of instantaneous values of voltages, the detection probability is described using the following relation:

$$
P D=1-\frac{1}{\sigma_{N} \sqrt{2 \pi}} \int_{x_{D}-x_{S}}^{\infty} \exp \left(\frac{-x^{2}}{2 \sigma_{N}^{2}}\right) d x,
$$

where $x_{D}$ is the value of a detection threshold and $\bar{x}_{S}$ is the average value of the signal.

Under the same conditions, the probability of a false alarm is expressed as

$$
P F A=\frac{1}{\sigma_{N} \sqrt{2 \pi}} \int_{x_{D}}^{\infty} \exp \left(\frac{-x^{2}}{2 \sigma_{N}^{2}}\right) d x .
$$

For low probability of false alarms and high probability of object detection when it is fulfilled, eqns (22) and (23) can be transformed into the following form:

$$
P D=1-\frac{\sigma_{N}}{\sqrt{2 \pi}\left(\bar{x}_{S}-x_{D}\right)} \exp \left(\frac{-\left(\bar{x}_{S}-x_{D}\right)^{2}}{2 \sigma_{N}^{2}}\right),
$$




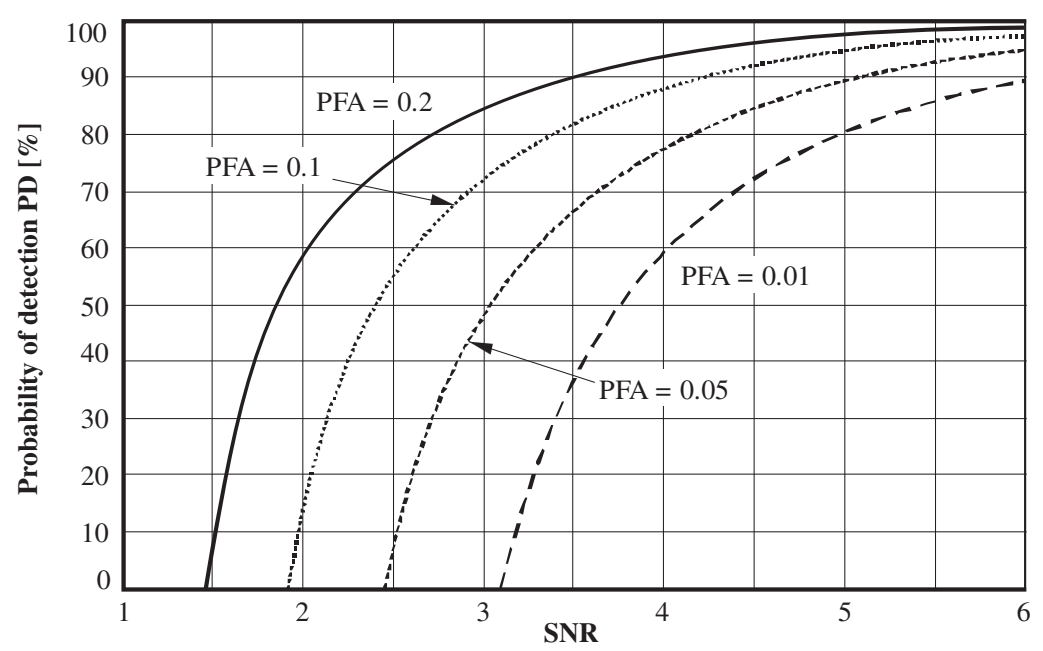

Figure 11: Probability of object detection for signal processed with the proposed analysis method.

$$
P F A=\frac{\sigma_{N}}{\sqrt{2 \pi} x_{D}} \exp \left(\frac{-x_{D}^{2}}{2 \sigma_{N}^{2}}\right) .
$$

These probabilities depend on two parameters, the ratio of a detection threshold value to the standard noise deviation $x_{D} / \sigma_{N}$ as well as the ratio of an average value of a signal to the standard noise deviation $\bar{x}_{S} / \sigma_{N}$.

The detection probability can be presented as a function of SNR by introducing the notation $S N R=\frac{\bar{x}_{S}}{\sigma_{N}}$ and transforming eqn (25) we have

$$
P D=1-\frac{1}{\sqrt{2 \pi\left(S N R-\frac{x_{D}}{\sigma_{N}}\right)}} \exp \frac{-\left(S N R-\frac{x_{D}}{\sigma_{N}}\right)^{2}}{2}
$$

For calculations of detection and false alarm probability, a standard noise deviation should be determined based on the changes in the $S_{k}(m)$ parameter, and a threshold value should be substituted instead of the $x_{D}$ value.

Figure 11 presents the diagrams of detection probability as a function of the SNR for a few assumed probabilities of a false alarm. According to eqn (26), the probability of a false alarm depends on the $x_{D} / \sigma_{N}$ ratio. In a method of signal analysis used for the PIR detector, one can take the higher value of $x_{D}$ (higher SNR). After filtration, a lower value of $\sigma_{N}$ is also obtained, which ensures lower values of false alarm probability. 


\section{CONCLUSION}

To recapitulate, it should be stated that the developed PIR detector detecting slowly moving and crawling people has the parameters allowing for its application in security systems. Satisfactory results of laboratory and field investigations of the developed and performed model of a PIR detector have confirmed that the developed detector detects the objects moving with the velocities from $5 \mathrm{~cm} / \mathrm{s}$ to $5 \mathrm{~m} / \mathrm{s}$. A detection range of crawling people (for the performed model) is of the order of 140-150 m. The method of signal analysis used for the PIR detector gives lower values of false alarm probability. The new method of signal processing gives a higher detection probability than PIR with normal signal processing.

\section{REFERENCES}

[1] Feng C. \& Xu P., The detection mechanism of LiTaO3 type II pyroelectric detectors. III. The total pyroelectric effects. Infrared Physics \& Technology, 40, pp. 79-82, 1999. doi: http://dx.doi.org/10.1016/S1350-4495(98)00044-9

[2] Yoshiike N., Morinaka K., Hashimoto K., Kawaguri M. \& Tanaka S., 360 direction type human information sensor. Sensor and Actuators, 77, pp. 199208, 1999. doi: http:// dx.doi.org/10.1016/S0924-4247(98)00367-7

[3] Kastek, M., Sosnowski, T., Polakowski, H., Dąbrowski, M. \& Orżanowski, T., Longrange PIR detector used for detection of crawling people. Proceedings of SPIE, 7113, pp. 71131F, 2008.

[4] ELTEC Instruments Inc., Catalogue of Firm Products, P.O. Box 9610, Central Business Park, Daytona Beach, Florida 32120-9610, USA, 1996.

[5] Kastek M., Sosnowski T. \& Piątkowski T., Passive infrared detector used for detection of very slowly moving of crawling people. Opto-Electronics Review, 16(3), pp. 328-335, 2008. doi: http://dx.doi.org/10.2478/s11772-008-0022-3

[6] Kastek, M., Madura, H., Morawski M., Piątkowski T., Powiada E. \& Polakowski H., Test bed for measurement of angular parameters of passive infrared sensors. Infrared Physics Technology, 49(3 SPEC. ISS.), pp. 198-201, 2007.

[7] Madura H., Method of signal processing in passive infrared detectors for security systems. WIT Transactions on Modeling and Simulation, 46, pp. 757-768, 2007. doi: http://dx.doi.org/10.2495/CMEM070741

[8] Madura H., Kastek M. \& Piatkowski T., Automatic compensation of emissivity in threewavelength pyrometers, Infrared Physics and Technology, 51(1), pp.1-8, 2007. doi: http://dx.doi.org/10.1016/j.infrared.2006.11.001

[9] Madura H. \& Kołodziejczyk M., Influence of sun radiation on results of non-contact temperature measurements in far infrared range. Opto-Electronics Review, 13(3), pp. 253-257, 2005.

[10] Madura H., Piątkowski T. \& Powiada E., Multispectral precise pyrometer for measurement of seawater surface temperature. Infrared Physics \& Technology, 46 pp. 69-73, 2004. doi: http://dx.doi.org/10.1016/j.infrared.2004.03.010

[11] Sosnowski, T., Madura, H., Kastek, M., Piątkowski T. \& Powiada E., Method of objects detection employing passive IR detectors for security systems. Proceedings of SPIE, 7113, pp. 71131G, 2008. doi: http://dx.doi.org/10.1117/12.800147

This paper has been selected for this special issue but first appeared in WIT Transactions on the Built Environment, Vol 108, (c) 2009 WIT Press, www.witpress.com, ISSN 1743-3509 (on-line), doi:10.2495/SAFE090071. 